\title{
New Differential-Algebraic Attacks and Reparametrization of Rainbow
}

\author{
Jintai Ding ${ }^{1}$, Bo-Yin Yang ${ }^{2}$, Chia-Hsin Owen Chen ${ }^{2}$, Ming-Shing Chen ${ }^{2}$, \\ and Chen-Mou Cheng ${ }^{3}$ \\ ${ }^{1}$ Dept. of Mathematical Sciences, University of Cincinnati, USA \\ ding@math.uc.edu \\ 2 IIS, Academia Sinica, Taiwan \\ \{byyang, owenhsin, mschen\}@iis.sinica.edu.tw \\ ${ }^{3}$ Dept. of Elec. Eng., Nat'l Taiwan University, Taiwan \\ ccheng@cc.ee.ntu.edu.tw
}

\begin{abstract}
A recently proposed class of multivariate Public-Key Cryptosystems, the Rainbow-Like Digital Signature Schemes, in which successive sets of central variables are obtained from previous ones by solving linear equations, seem to lead to efficient schemes (TTS, TRMS, and Rainbow) that perform well on systems of low computational resources. Recently SFLASH $\left(\mathrm{C}^{*-}\right)$ was broken by Dubois, Fouque, Shamir, and Stern via a differential attack. In this paper, we exhibit similar algebraic and diffential attacks, that will reduce published Rainbow-like schemes below their security levels. We will also discuss how parameters for Rainbow and TTS schemes should be chosen for practical applications.
\end{abstract}

Keywords: rank, differential attack, algebraic attack, oil-and-vinegar.

Note: Up-to-date version will be available at eprint . iacr .org/2008/108

\section{Outline}

Multivariate Public-Key Cryptosystems (MPKCs, or trapdoor $\mathcal{M} \mathcal{Q}$ schemes) are cryptosystems for which the public key is a set of polynomials $\mathcal{P}=\left(p_{1}, \ldots, p_{m}\right)$ in variables $\mathbf{x}=\left(x_{1}, \ldots, x_{n}\right)$ where all variables and coefficients are in $\mathbb{K}=$ $\mathrm{GF}(q)$. In practice this is always accomplished via

$\mathcal{P}: \mathbf{w}=\left(w_{1}, \ldots, w_{n}\right) \in \mathbb{K}^{n} \stackrel{S}{\mapsto} \mathbf{x}=\mathrm{M}_{S} \mathbf{w}+\mathbf{c}_{S} \stackrel{\mathcal{Q}}{\mapsto} \mathbf{y} \stackrel{T}{\mapsto} \mathbf{z}=\mathrm{M}_{T} \mathbf{y}+\mathbf{c}_{T}=\left(z_{1}, \ldots, z_{m}\right) \in \mathbb{K}^{m}$

In any given scheme, the central map $\mathcal{Q}$ belongs to a certain class of quadratic maps whose inverse can be computed relatively easily. The maps $S, T$ are affine. The polynomials giving $y_{i}$ in $\mathbf{x}$ are called the central polynomials, and the $x_{j}$ are called the central variables.

In 1999, the Unbalanced Oil-and-Vinegar multivariate structure is proposed by Patarin et al [16]. Lately the Rainbow class of signatures [7, 25, 20], based on 
repeated applications of the Unbalanced Oil-and-Vinegar principle, shows some promise on systems of low computational resources.

Given that the well-known $C^{*-}$ class of signature schemes including SFLASH was broken by differential attacks [8], we examine similar attacks on Rainbow, with the following conclusions:

- Differentials improve on the High-Rank attacks on Rainbow-like systems.

- Differentials also helps with randomized brute-force searches for $S$ and $T$.

- We can assess how Rainbow-like schemes needs to be amended in view of recent developments.

- The results are in line with experiments run on small scale systems.

In Sec. 2 we recap Rainbow-like multivariates and what is known about the security of MPKC before the appearance of Rainbow in Sec. 3 . In Sec. 4, we describe the new differential attack, which is related to the high-rank attack, and in Sec.5 we present new paramters for Rainbow construction, we tabulate what we know about the security of Rainbow-like schemes, in particular, the security against the two new recent attacks specially targeted against the Rainbow schemes, and we design schemes with new parameters for practical applications. Finally, in Sec. 6, we present the conclusion.

\section{Rainbow-Like Multivariate Signatures}

We characterize a Rainbow type PKC with $u$ stages:

- The segment structure is given by a sequence $0<v_{1}<v_{2}<\cdots<v_{u+1}=n$. For $l=1, \ldots, u+1$, set $S_{l}:=\left\{1,2, \ldots, v_{l}\right\}$ so that $\left|S_{l}\right|=v_{l}$ and $S_{0} \subset S_{1} \subset$ $\cdots \subset S_{u+1}=S$.

- Denote by $o_{l}:=v_{l+1}-v_{l}$ and $O_{l}:=S_{l+1} \backslash S_{l}$ (i.e., $v_{l}<k \leq v_{l+1}$ if $k \in O_{l}$ ) for $l=1 \cdots u$. The central map $\mathcal{Q}: \mathbf{x}=\left(x_{1}, \ldots, x_{n}\right) \mapsto \mathbf{y}=\left(y_{v_{1}+1}, \ldots, y_{n}\right)$, where each $y_{i}:=q_{i}(\mathbf{x})$ is a quadratic polynomial in $\mathbf{x}$ of the following form

$$
q_{k}=\sum_{i<j \leq v_{l}} \alpha_{i j}^{(k)} x_{i} x_{j}+\sum_{i \leq v_{l}<j<v_{l+1}} \alpha_{i j}^{(k)} x_{i} x_{j}+\sum_{i<v_{l+1}} \beta_{i}^{(k)} x_{i}, \quad \text { if } v_{l}<k \leq v_{l+1} .
$$

In every $q_{k}, k \in O_{l}$, there is no cross-term $x_{i} x_{j}$ where both $i$ and $j$ are in $O_{l}$ at all. So given all the $y_{i}$ with $v_{l}<i \leq v_{l+1}$, and all the $x_{j}$ with $j \leq v_{l}$, we can compute $x_{v_{l}+1}, \ldots, x_{v_{l+1}}$.

- To expedite computations, some coefficients $\alpha_{i j k}$ 's may be fixed (e.g., set to zero), chosen at random (and included in the private key), or be interrelated in a predetermined manner.

- To invert $\mathcal{Q}$, determine (usu. at random) $x_{1}, \ldots x_{v_{1}}$, i.e., all $x_{k}, k \in S_{1}$. From the components of $\mathbf{y}$ that corresponds to the polynomials $q_{v_{1}+1}, \ldots q_{v_{2}}$, we obtain a set of $o_{1}$ equations in the variables $x_{k},\left(k \in O_{1}\right)$. We may repeat the process to find all remaining variables. 
In this form, we can see that Rainbow can only be a signature scheme. We can see a good example of what can go wrong in [15] if we try to construct an encryption scheme, where the initial vinegar variables is determined through an initial block of equations.

Example 1. enTTS $(20,28)$ of [25] has structure $(8,9,1,1,9)$ and this central map:

$$
\begin{aligned}
y_{i}=x_{i}+ & \sum_{j=1}^{7} p_{i j} x_{j} x_{8+(i+j \bmod 9)}, i=8 \cdots 16 \\
y_{17}=x_{17} & +p_{17,1} x_{1} x_{6}+p_{17,2} x_{2} x_{5}+p_{17,3} x_{3} x_{4} \\
& +p_{17,4} x_{9} x_{16}+p_{17,5} x_{10} x_{15}+p_{17,6} x_{11} x_{14}+p_{17,7} x_{12} x_{13} ; \\
y_{18}=x_{18} & +p_{18,1} x_{2} x_{7}+p_{18,2} x_{3} x_{6}+p_{18,3} x_{4} x_{5} \\
& +p_{18,4} x_{10} x_{17}+p_{18,5} x_{11} x_{16}+p_{18,6} x_{12} x_{15}+p_{18,7} x_{13} x_{14} ; \\
y_{i}=x_{i}+ & p_{i, 0} x_{i-11} x_{i-9}+\sum_{j=19}^{i-1} p_{i, j-18} x_{2(i-j)-(i \bmod 2)} x_{j}+p_{i, i-18} x_{0} x_{i} \\
& +\sum_{j=i+1}^{27} p_{i, j-18} x_{i-j+19} x_{j}, i=19 \cdots 27 .
\end{aligned}
$$

If $x_{0}, \ldots, x_{7}$ is decided, one can solve first for $x_{8}, \ldots, x_{16}$, then $x_{17}, x_{18}$, then $x_{19}, \ldots, x_{27}$. Note: $x_{0}$ does not appear until the last block, which will be significant later.

Example 2. The proposed Rainbow scheme in [7] is an essentially generic stagewise UOV construction with layers $(6,6,5,5,11)$. The first six central equations is a generic UOV construction with six vinegar $\left(x_{1}, \ldots x_{6}\right)$ and six oil $\left(x_{7}, \ldots, x_{12}\right)$ variables; the next five has 12 vinegars and 5 oils $\left(x_{13}, \ldots, x_{17}\right)$; the next five has 17 vinegars and 5 oils $\left(x_{18}, \ldots, x_{22}\right)$, and the last 11 has 22 vinegars and 11 oils $\left(x_{23}, \ldots, x_{33}\right)$.

Rainbow schemes where most of the crossterm coefficients $\alpha_{i j}^{(k)}$ are zero are said to be TTS instances. TTS schemes have a relatively small private key and even better efficiency, but may be exposed to additional risks. Regardless, the same techniques that we shall describe below are security concerns for all schemes of the rainbow type including TTS, TRMS, and Rainbow [25, 20, 7].

\section{The Security of Multivariates and Prior Attacks}

The name of the class came from the "Multivariate Quadratics" problem:

Problem $\mathcal{M Q}$ : Solve the system $p_{1}=p_{2}=\cdots=p_{m}=0$, where each $p_{i}$ is a quadratic polynomial in $\mathbf{x}=\left(x_{1}, \ldots, x_{n}\right)$ and coefficients and variables are in $\mathbb{K}=\mathrm{GF}(q)$.

Generic $\mathcal{M Q}$ is NP-hard [12, and consensus pegs it as a difficult problem to solve even probabilistically. However, to use $\mathcal{M Q}$ as the underlying hard problem in a $\mathrm{PKC}$, one need a trapdoor built into the public map $\mathcal{P}$. So the security of the cryptosystem also depends on the following:

Problem EIP: (Extended Isomorphism of Polynomials) Given a class of central maps $\mathfrak{C}$ and a map $\mathcal{P}$ expressible as $\mathcal{P}=T \circ \mathcal{Q} \circ S$, where $\mathcal{Q} \in \mathfrak{C}$, and $S, T$ are affine, make such a decomposition. 
There are two interesting twists here:

- If $\mathcal{Q}$ is constant, this is known as the IP problem. J.-C. Faugère showed that in some cases simple IP is not NP-hard at Eurocrypt 2006 [11.

- The EIP problem where $\mathfrak{C}$ is the set of homogeneous quadratic maps is easy [13]. Equivalently, if $\mathcal{Q}$ is homogeneous (e.g., as in $\mathrm{SFLASH}=C^{*-}$ ) we can set $\mathbf{c}_{S}=\mathbf{c}_{T}=0$.

If $\mathcal{Q}$ fundamentally involves a map in a field $\mathbb{L}=\mathbb{K}^{k}$ that is of a size significantly bigger than $\mathbb{K}$, we call the scheme "big field" or "dual field". This order includes derivatives of Matsumoto-Imai $\left(C^{*}\right)$ and Hidden Field Equations. Otherwise we call the scheme a "true multivariate" (sometimes "single field"). This includes the Unbalanced Oil-and-Vinegar and stagewise triangular structures.

One of the biggest concerns of multivariate cryptography is the lack of provable security results. Today security in MPKC is still very much ad hoc. Proposed schemes are evaluated against known attacks security estimates obtained for various parameters. The designers then tries to juggle the system parameters so as to have some requisite security level under every known attack.

With that, we list the standard attacks known for MPKCs today:

1. Rank (or Low Rank, MinRank) attack, which finds a central equation with least rank [25].

$$
C_{\text {low rank }} \approx\left[q^{r\lceil m / n\rceil} m\left(n^{2} / 2-m^{2} / 6\right) / \mu\right] \mathfrak{m} .
$$

Here as below, the unit $\mathfrak{m}$ is a multiplications in $\mathbb{K}$, and $r$ is that lowest rank ("MinRank", [14). $\mu$ is the number of linear combinations of central equations [25] at that minimal rank.

2. Dual Rank (or High Rank) attack [5, 14], which finds a variable appearing the fewest number of times in a central equation cross-term. If this least number is $s$, 25] gives

$$
C_{\text {high rank }} \approx\left[q^{s} n^{3} / 6\right] \mathfrak{m} .
$$

3. Oil-and-Vinegar Separation [22, 16, 17, which finds an Oil subspace that is sufficiently large (estimates as corrected in [25]).

$$
C_{\mathrm{UOV}} \approx\left[q^{n-2 o-1} o^{4}+\left(\text { some residual term bounded by } o^{3} q^{m-o} / 3\right)\right] \mathfrak{m} .
$$

$o$ is the max. oil set size, i.e., there is a set of $o$ central variables which are never multiplied together in the central equations, and no more.

4. Trying for a direct solution (i.e., going for the $\mathcal{M Q}$ as opposed to the EIP or "structural" problem). Best known methods are the Lazard-Faugère family of solvers (the Gröbner Bases methods $\mathbf{F}_{\mathbf{4}}-\mathbf{F}_{\mathbf{5}}$ or XL) whose complexities 6, 9, 10, 24 are very hard to evaluate; some recent asymptotic formulas can be found in [1,2,24]. 


\section{New Differential Attacks}

One key point of our new attack is to use the differentials (first used, as far as we know, with MPKC in [18, and recently to break SFLASH [8]).

Given the public key of a MPKC, which we denote as $\mathcal{P}(\mathbf{x})$, a set of quadratic polynomials, its differential $D \mathcal{P}(\mathbf{x})$ is defined as

$$
D \mathcal{P}(\mathbf{x})=\mathcal{P}(\mathbf{x}+\mathbf{c})-\mathcal{P}(\mathbf{x})-\mathcal{P}(\mathbf{c}),
$$

a set of linear functions in $\mathbf{x}$.

The key is to use the hidden structures in the differential to attack the cryptosystem. The observation is that the differential can be used to improve the old high-rank attack when there are too many variables that don't appear in the final block of equations (for $y_{i}$, where $i \in O_{u}$ ). First, we will reformulate an existing attack in terms of the differentials.

Let $H_{i}$ be the symmetric matrix corresponding to the quadratic part of $z_{i}(\mathbf{w})$. Without loss of generality, we may let the fewest number of appearances of all variables in the cross-terms of the central equations be the last variable $x_{n}$ appearing $s$ times.

Algorithm 0 (High or Dual Rank Attack). as described by Goubin-Courtois and Yang-Chen [14,25]:

1. Compute the differential $\mathcal{P}(\mathbf{x}+\mathbf{c})-\mathcal{P}(\mathbf{x})-\mathcal{P}(\mathbf{c})$ and take its $j$-th component (which is bilinear in $\mathbf{x}$ and $\mathbf{c}$ ) as $\mathbf{c}^{T} H_{j} \mathbf{x} . H_{k}$ is representing the quadratic crossterms in the $k$-th polynomial of the public key. Note that the $H_{i}$ are always symmetric and if char $\mathbb{K}=2$, and $\mathbf{x}^{T} H_{i} \mathbf{x}=0$.

2. Form an arbitrary linear combination $H=\sum_{i} \alpha_{i} H_{i}$. Find $V=\operatorname{ker} H$.

3. When $\operatorname{dim} V=1$, set $\left(\sum_{j} \lambda_{j} H_{j}\right) V=\{\mathbf{0}\}$ and check if the solution set $\hat{V}$ of the $\left(\lambda_{i}\right)$ form a subspace dimension $m-s$. Note: Since a matrix in $K^{n \times n}$ can have at most $n$ different eigenvalues, less than $n / q$ of the time we would need to do this.

4. With probability $q^{-s}$ we have $V=U=\left\{\mathbf{x}: x_{1}=\cdots=x_{v_{u}}=0\right\}$.

As each trial run consists of running an elimination and some testing, we can realistically do this with $\sim\left(s n^{2}+\frac{n^{3}}{6}\right) q^{s}$ field multiplications, by taking linear combinations from only $(s+1)$ of the matrices $H_{i}$ and hope not to get too unlucky. An upper bound is $\left[m n^{2}+\frac{n^{3}}{6}+\frac{n}{q}\left(m^{3} / 3+m n^{2}\right)\right] q^{s}$.

The above formulation of the high rank attack is designed to defeat "plus"modified Triangular systems. We first present some notations before describing how we can improve this attack further:

Let $P_{l}$ be the linear space of quadratic polynomials spanned by polynomials of the form

$$
\sum_{i \in O_{l}, j \in S_{l}} \alpha_{i, j} x_{i} x_{j}+\sum_{i, j \in S_{l}} \alpha_{i, j} x_{i} x_{j}+\sum_{i \in S_{l+1}} \beta_{i} x_{i}+\eta
$$


We can see that these are Oil and Vinegar type of polynomials such that $x_{i}$, $i \in O_{l}$ are the Oil variables and $x_{i}, i \in S_{l}$ are the Vinegar variables. We call $x_{i}$, $i \in O_{l}$ an $l$-th layer Oil variable and $x_{i}, i \in S_{l}$ an $l$-th layer Vinegar variable. We call any polynomial in $P_{l}$ an $l$-th layer Oil and Vinegar polynomial. Clearly we have $P_{i} \subset P_{j}$ for $i<j$. Let $W_{i}$ be the space of linear functions of variables $x_{1}, \ldots, x_{v_{i}}$. Then we have

$$
W_{1} \subset P_{1} \subset W_{2} \subset P_{2} \cdots \subset W_{u} \subset P_{u} \subset W_{u+1}
$$

Now we present the new attack:

Algorithm 1. The Improved High-Rank Attack using differentials:

1. Pick random $\mathbf{c}, \mathbf{c}^{\prime} \in \mathbb{K}^{n}$, compute $\mathcal{P}(\mathbf{w}+\mathbf{c})-\mathcal{P}(\mathbf{w})-\mathcal{P}(\mathbf{c})$, and we will denote its components as $\left(t_{1}, t_{2}, \ldots, t_{m}\right)$. Similarly we compute $\left(t_{1}^{\prime}, t_{2}^{\prime}, \ldots, t_{m}^{\prime}\right)=$ $\mathcal{P}\left(\mathbf{w}+\mathbf{c}^{\prime}\right)-\mathcal{P}(\mathbf{w})-\mathcal{P}\left(\mathbf{c}^{\prime}\right)$, then

$$
U=\operatorname{span}\left(t_{1}, t_{2}, \ldots, t_{m}\right) \cap \operatorname{span}\left(t_{1}^{\prime}, t_{2}^{\prime}, \ldots, t_{m}^{\prime}\right) .
$$

2. Guess at a linear form $f \in U$; find coefficients $a_{i}$ and $a_{i}^{\prime}$ such that $f=$ $\sum a_{i} t_{i}=\sum a_{i}^{\prime} t_{i}^{\prime}$

3. Use $a_{i}$ and $a_{i}^{\prime}$ as the guessed $\alpha_{i}$ in the High Rank Attack (Algorithm Q) above.

Proposition 1. The expected complexity of Algorithm 1 is $\sim q^{d}$. (cubic-time elimination) where (the last block of equations is the ones whose solutions gives $\mathrm{O}_{u}$ )

$$
d \leq s-[\# \text { vars appearing in crossterms only in the last block]. }
$$

Proof. Let

$$
F=\left(F_{1}, \ldots, F_{m}\right)=\mathcal{Q} \circ S
$$

be the mapping from $\mathbf{x} \mapsto \mathbf{z}$. Let

$$
F(\mathbf{x}+\mathbf{b})-F(\mathbf{x})-F(\mathbf{b}):=G=\left(G_{1}, G_{2}, \ldots, G_{n}\right),
$$

where $\mathbf{b}=\left(b_{1}, b_{2}, \ldots b_{i}, \ldots, b_{n}\right)$ is randomly chosen. Pick another $\mathbf{b}^{\prime}$ and form

$$
H=\left(H_{1}, \ldots, H_{n}\right)=F\left(\mathbf{x}+\mathbf{b}^{\prime}\right)-F(\mathbf{x})-F\left(\mathbf{b}^{\prime}\right),
$$

then

1. if $i \in O_{j}$, then $G_{i}, H_{i} \in W_{j+1}$;

2. $\bar{W}_{j+1}:=\operatorname{span}\left\{G_{i}\right\}_{i \in O_{j}} \subset W_{j+1}$, and similarly $\widehat{W}_{j+1}:=\operatorname{span}\left\{H_{i}\right\}_{i \in O_{j}} \subset$ $W_{j+1}$

3. $\bar{W}_{2} \subset \ldots \subset \bar{W}_{u+1}$ and $\widehat{W}_{2} \subset \ldots \subset \widehat{W}_{u+1}$.

Clearly $\left(\widehat{W}_{u} \cap \bar{W}_{u}\right) \subset\left(\widehat{W}_{u+1} \cap \bar{W}_{u+1}\right)$, and we observe that: if the dimensions of the two subspaces differ by $d$, then we can break the system with $\propto q^{d}$. (one guess) computations. 
How so? Because the relationship between $\mathcal{P}$ and $F$, is the same as that between the $\mathbf{w}$-space and $\mathbf{x}$-space, i.e., the linear transformation $S$. So there is a 1-in- $q^{d}$ chance that both $\sum a_{i} z_{i}$ and $\sum a_{i}^{\prime} z_{i}$ correspond to a linear form in $W_{u}$. The odds are now decided by $q^{-d}$ instead of $q^{-s}$. In a Rainbow-like system, $s=o_{u}=n-v_{u}$. For Alg. 1 to be worthwhile, we must show that $d \leq s$.

In fact, it is not so hard to describe how to determine $d . \bar{W}_{u+1}$ and $\widehat{W}_{u+1}$ are two $m$-dimensional subspaces in the $n$-dimensional vector space $W_{u+1}$. Most of the time they intersect in a $2 m-n$ dimensional subspace, hence

$$
\operatorname{dim} \bar{W}_{u}=\operatorname{dim} \widehat{W}_{u}=m-o_{u}
$$

which equals the number of variables appearing in cross-terms in equations not of the final block, which is equivalent to Eq. 2.

Example 3. Consider enTTS $(20,28)$ as in Eq. 1. Here $\operatorname{dim}\left(\bar{W}_{u+1} \bigcup \widehat{W}_{u+1}\right)=$ $20+20-28=12$, while $\operatorname{dim}\left(\bar{W}_{u} \cup \widehat{W}_{u}\right)=11+11-17=5$. Therefore we need only $\sim 2^{56}$ instead of $2^{72}$ guesses, which is a speed increase of $2^{16} \times$ over Algorithm 0. Since each guess takes about $2^{8}$ time units (standard is to use time of a 3DES block encryption, between $2^{6}$ to $2^{8}$ multiplications), this gives complexity $2^{64}$ instead of $2^{80}$, too weak to be "strong" crypto.

What went wrong? Generically $\operatorname{dim} W_{u}=n-o_{u}$ and the intersection is of dimension $2\left(m-o_{u}\right)-\left(n-o_{u}\right)=2 m-n-o_{u}$, making $d=(2 m-n)-(2 m-$ $\left.n-o_{u}\right)=o_{u}=s$. The lesson: watch out for variable not in the final oil set that does not occur prior to the last block of equations. In enTTS $(20,28), x_{0}$ and $x_{18}$ did not appear in any earlier equations than the final block.

\subsection{Experimentation with Mini-versions}

We experimented in smaller fields with three different schemes: Rainbow $(6,6,5,5$, $11)$, the enTTS $(20,28)$ scheme above, and its miniaturized sister version enTTS $(16,22)$ [structure $(6,7,1,1,7)]$.

Table 1. Timing (sec) on 16 of $3 \mathrm{GHz}$ P4 machines guessing in parallel

\begin{tabular}{|c|c|r|r|r|r|}
\hline Scheme & Structure & $q$ & Alg. 0 & Alg. 1 & ratio \\
EnTTS(20,28) & $(8,9,1,1,9)$ & 8 & 93 & 4.4 & 0.047 \\
EnTTS(20,28) & $(8,9,1,1,9)$ & 16 & 42435 & 496 & 0.012 \\
EnTTS(16,22) & $(6,7,1,1,7)$ & 16 & 102 & 3.5 & 0.034 \\
Rainbow [7] & $(6,6,5,5,11)$ & 8 & 8454 & 17123 & 2.028 \\
\hline
\end{tabular}

The results are fairly constant over many tests [except the enTTS(20,28) test which we only ran a few times]. Clearly, not having all vinegar variables of the last segment appearing previously in cross-terms is a big minus. Rainbow $(6,6,5,5,11)$ does not have the same problem and Algorithm 1 is no improvement of the High Rank Attack against it. 


\section{New Rainbow Parameters for Practical Applications}

For practical applications, we will propose the following Rainbow Structures.

1. $(20,10,4,10)$, where the public key has 44 variables and 24 polynomials.

2. $(18,12,12)$, where the public key has 42 variables and 24 polynomials.

3 . $(20,14,14)$, where the public key has 48 variables and 28 polynomials.

We will first formalize a twist on the regular Rainbow construction, which is somewhat more general. In the previous constructions, in each new layer, previously appeared variables will only be Vinegar variables, the new variables appearing only as Oil variables. We can also consider adding new Vinegar variables as we add Oil variables. This also implies that in the signing process, we guess at the new vinegar variables as they appear, while in the previous Rainbow construction, we only guess the Vinegar variables in the first layer once. In this case, we can also write for each layer two parameters, $\left(v_{i}^{\prime}, o_{i}\right)$, where the $v_{i}^{\prime}$ counts the new vinegar variables we introduce. In this layer, we will have $v_{i}+v_{i}^{\prime}$ Vinegar variables (where $v_{i}$ counts the number of all previous appearing variables) and $o_{i}$ the number of Oil variables.

If all the $v_{i}^{\prime}$ are zero, this is precisely the original Rainbow construction. We might call this new construction the extended Oil-Vinegar construction. From the viewpoint of the attacker we can see this as a specialization of the Rainbow construction, since the new vinegar variables might as well have been part of the initial block of vinegar variables, but simply never have been used before. However, it is different in an operative sense, in that if we use the new vinegar variables properly, we could always find a signature, as implicitly used in TTS constructions earlier.

So, in this language, we would propose scheme: $((15,10),(4,4),(1,10)),((17,12)$, $(1,12))$, and $((19,14),(1,14))$.

For these new schemes, we could also choose to use the generic sparse polynomials or special sparse polynomials as in the case of TTS 25]. For generic sparse polynomials, we think it is a good idea to choose $3 L_{i}$ terms for each layer, where $L_{i}$ is the sum of number of Oil and vinegar variables in each layer.

For these new schemes, we need to take into two new recent special attacks against Rainbow.

\subsection{The Reconciliation Attack}

In the following attack we attempt to find a sequence of change of basis that let us invert the public map. In this sense it can be considered an improved brute force attack.

Suppose we have an oil-and-vinegar structure, then the quadratic part of each component $q_{i}$ in the central map from $\mathbf{x}$ to $\mathbf{y}$, when expressed as a symmetric matrix, looks like 


$$
M_{i}:=\left[\begin{array}{ccc|ccc}
\alpha_{11}^{(i)} & \cdots & \alpha_{1 v}^{(i)} & \alpha_{1, v+1,}^{(i)} & \cdots & \alpha_{1 n}^{(i)} \\
\vdots & \ddots & \vdots & \vdots & \ddots & \vdots \\
\alpha_{v 1}^{(i)} & \cdots & \alpha_{v v}^{(i)} & \alpha_{v, v+1}^{(i)} & \cdots & \alpha_{v n}^{(i)} \\
\hline \alpha_{v+1,1}^{(i)} & \cdots & \alpha_{v+1, v}^{(i)} & 0 & \cdots & 0 \\
\vdots & \ddots & \vdots & \vdots & \ddots & \vdots \\
\alpha_{n 1}^{(i)} & \cdots & \alpha_{n v}^{(i)} & 0 & \cdots & 0
\end{array}\right]
$$

First, no matter what $\mathrm{M}_{T}$ is, it won't change the basic shape, so we let $T$ be the identity map for the moment. What can $S$ be like? Suppose we pick $\mathrm{M}_{S}$ as totally random, most often (see below) it decompose to

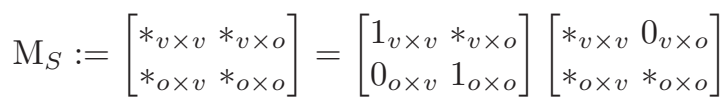

where 1 means identity matrix, 0 means just zeros and $*$ means random or anything. In fact, this decomposition always hold unless the lower-right $o \times o$ submatrix is singular. It should be clear that the $\left[\begin{array}{l}*_{v \times v} \\ *_{o \times v} *_{o \times o}\end{array}\right]$ portion of $\mathrm{M}_{S}$, as a coordinate change leaves the $M_{i}$ 's with the same shape. That is, if we can find the correct $\left[\begin{array}{ll}1_{v \times v} *_{v \times o} \\ 0_{o \times v} & 1_{o \times o}\end{array}\right]$ portion and perform the basis change in reverse, we will again make the resulting public map into the same form (all zeroes on the lower right) and be easily inverted. Hence, no more security at all. More about this phenomenon ("equivalent keys") in MPKCs can be seen in, say, [23.

Let this essential portion of $\mathrm{M}_{S}$ that we wish to recreate be $P$, that is, the linear transformation $\mathbf{w} \mapsto \mathbf{x}=P \mathbf{w}$ will create all zeroes on the lower right. We can decompose this $P$ into a product of $P:=P_{v+1} P_{v+2} \cdots P_{n}$, where each of the matrices look like

$$
P_{n}=1_{n}+\left[\begin{array}{ccc|c}
0 & \cdots & 0 & a_{1} \\
0 & \cdots & 0 & a_{2} \\
\vdots & \ddots & \vdots & \vdots \\
0 & \cdots & 0 & a_{v} \\
\hline 0 & \cdots & 0 & 0 \\
\vdots & \ddots & \vdots & \vdots \\
0 & \cdots & 0 & 0
\end{array}\right] ; \quad P_{n-1}=1_{n}+\left[\begin{array}{ccc|c|c}
0 & \cdots & 0 & a_{1}^{\prime} & 0 \\
0 & \cdots & 0 & a_{2}^{\prime} & 0 \\
\vdots & \ddots & \vdots & \vdots & \vdots \\
0 & \cdots & 0 & a_{v}^{\prime} & 0 \\
\hline 0 & \cdots & 0 & 0 & 0 \\
\vdots & \ddots & \vdots & \vdots & \vdots \\
0 & \cdots & 0 & 0 & 0
\end{array}\right] ; \cdots
$$

Indeed, the multiplication is actually commutative among the various $P_{i}$ 's. Suppose, then, that we start with the differential matrices $H_{i}$ and simultaneously transform them to make their lower-right corner a square of 0 's using exactly such $P_{i}$ 's.

Algorithm 2 (UOV Reconciliation). The following gives the Reconciliation Attack against a UOV scheme with o oil and $v=n-o$ vinegar variables (which has the smaller indices): 
1. Perform basis change $w_{i}:=w_{i}^{\prime}-\lambda_{i} w_{n}^{\prime}$ for $i=1 \cdots v, w_{i}=w_{i}^{\prime}$ for $i=$ $v+1 \cdots n$. Evaluate $\mathbf{z}$ in $\mathbf{w}^{\prime}$.

2. Let all coefficients of $\left(w_{n}^{\prime}\right)^{2}$ be zero and solve for the $\lambda_{i}$. We may use any method such as $\mathbf{F}_{\mathbf{4}} / \mathbf{F}_{\mathbf{5}}$ or FXL. There will be $m$ equations in $v$ unknowns.

3. Repeat the process to find $P_{n-1}$. Now we set $w_{i}^{\prime}:=w_{i}^{\prime \prime}-\lambda_{i} w_{n-1}^{\prime \prime}$ for $i=1 \cdots v$, and set every $\left(w_{n-1}^{\prime \prime}\right)^{2}$ and $w_{n}^{\prime \prime} w_{n-1}^{\prime \prime}$ term to zero (i.e., more equations in the system) after making the substitution. This time there are $2 m$ equations in $v$ unknowns.

4. Continue similarly to find $P_{n-2}, \ldots, P_{v+1}$ with more and more equations.

Given what we know about system-solving today, we can expect the complexity to be determined in solving the initial system. Hence, if $v<m$, solving $m$ equations in $v$ variables will be easier than $m$ equations in $n$ equations, and we achieve a simplification.

Proposition 2. The Reconciliation Attack works with probability $\approx\left(1-\frac{1}{q-1}\right)$.

Proof (Sketch). Provided that lower-right $o \times o$ submatrix of $\mathrm{M}_{S}$ is non-singular, we can see that the construction of $P_{n}$ will eliminate the quadratic term in the last variable. $P_{n-1}$ will eliminate all quadratic terms in the last two variables, and so on, and each sequential construction will not disturb the structure built by the prior transformations. The number of nonsingular $k \times k$ matrices in over $\operatorname{GF}(q)$ is $\left(q^{k}-1\right)\left(q^{k}-q\right)\left(q^{k}-q^{2}\right) \cdots\left(q^{k}-q^{k-1}\right)$, because the first row has 1 possibility to be zero, the second row $q$ possibilities to be a multiple of the first, the third row $q^{2}$ possibilities to be dependent on the first two, etc., so the chance that the above attack works is roughly

$$
\left(1-\frac{1}{q}\right)\left(1-\frac{1}{q^{2}}\right) \cdots\left(1-\frac{1}{q^{k}}\right)>1-\left(\frac{1}{q}+\frac{1}{q^{2}}+\cdots+\frac{1}{q^{k}}\right)>1-\frac{1}{q-1} .
$$

Here we will use formulas from [26] for all our estimates as shown below.

Example 4. We attack enTTS $(20,28)$ as in Eq. 1. Originally we must solve a 20equation, 20-variable (we can guess 8 out of the original 28) MQ system. With $v_{u}=19$, the rate-determining step of the Reconciliation Attack is a 20-equation, 19 -variable system. This is easier by a factor of exactly $2^{8}$ if we are using FXL or $\mathrm{FF}_{4}$ [24, 1], since we will guess exactly one fewer variable.

Since we expect a direct attack on enTTS(20,28) to have $\sim 2^{72}$ complexity, Alg. 2 should take $\sim 2^{64}$. The construction process and odds as given above have been tested and verified on miniature versions (cf. 25) of TTS schemes such as enTTS $(16,22)$ as well as other Rainbow-like instances.

Example 5. TRMS [20] can be reduced to $2^{64}$ via the same attack (a faster attack given below) because it has rainbow layer parameters of $(8,6,2,3,9)$, with a last block of the same size as TTS.

Example 6. We implemented enTTS(16,22) over GF(128), the initial system has 16 equations and $22-7=15$ variables. We ran FXL with Wiedemann solver 
(as in [26]) with one fixed variable on an assembly of machines with 128 total $\mathrm{P} 4$ cores at $3.0 \mathrm{GHz}$, each guessing 1 value out of 128 . Here $D=8$ [24], and the number of monomials is $T=319770$, with a total of 73799040 terms which took only $288 \mathrm{MB}$ of storage at every core. Solving a system known to have a solution should take around $3\left(T^{2} n(n+3) / 2\right) \approx 2^{45}$ multiplications, which at about 16 cycles a multiplication about $2.0 \times 10^{4}$ seconds, but we discovered that there is guesswork in generating a system, so we dare not run more than one value on a given CPU.

In practice we were not so unlucky and were able to solve 15 variables in 16 equations in GF(128) in what was in fact closer about 3 days, probably due to non-optimal programming. After that, solving the remaining systems is a piece of cake [real CPU time estimated at less than two hours], and we can then decompose an enTTS(16,22) instance.

Example 7. We now attack the proposed Rainbow instance in [7. Since $v_{u}=$ $22<m=26$, solving this one is significantly easier: using $\mathrm{FF}_{\mathbf{5}}$ 24, the expected time use is $2^{56}$ (3DES blocks) instead of $2^{81} . \mathbf{F}_{\mathbf{5}}$ is not generally available but we should be able to achieve $\sim 2^{64}$ cycles using FXL on a large SMP system.

We can easily see that we must be very careful choosing our parameters for security against one attack may expose it to another. Our selected parameters are all tuned against this particular attack and this attack is no better or worse than direct attack, which have complexity of solving 24, and 28 equations in as many variables over $\mathrm{GF}(256)$, or roughly $2^{83}$ and $2^{98}$ respectively.

But this is just a unbalanced oil and vinegar attack. The more efficiently implemented systems are Rainbow and have multiple layers. If we look at the Rainbow construction, it looks more like

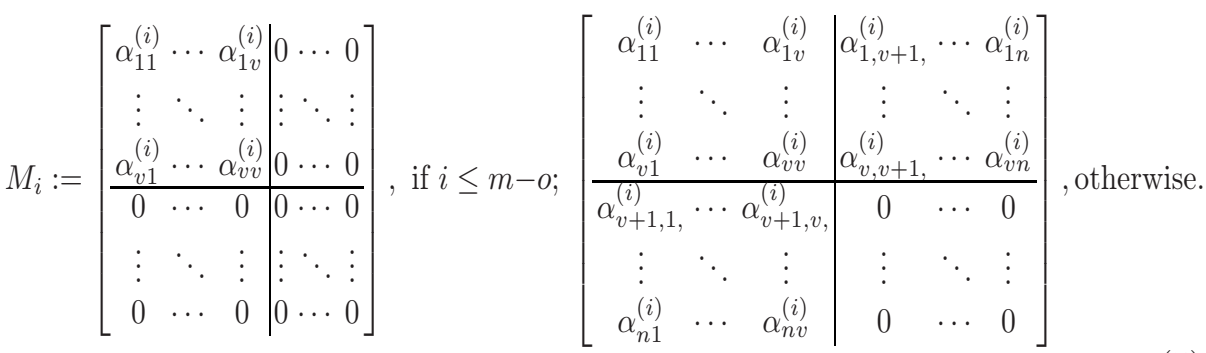

That is, only the last $o$ equations looks like Eq. 3, the initial $m-o$ equations actually have non-zero entries in the upperleft submatrix — which actually looks like a UOV matrix itself, i.e., has a block of zeros on the lower right. We don't bother with that detail. Can we exploit this property? Yes we can.

At this point, we should no longer consider $T$ as the identity. Let us think about what the matrix $\mathrm{M}_{T}$ does in Rainbow. At the moment that we distill the $P_{n}$ portion out, $m-o$ of the new $M_{i}$ 's should show a zero last column. However we don't: $\mathrm{M}_{T}$ mixes the $M_{i}$ 's together so that they in fact don't - we will see most of the time only the lower right entry as zero. But if we take any o +1 of those last columns, there will be a non-trivial linear dependency. We can verify 
that by setting one of those columns as the linear combination as the other o, the resulting equations are still quadratic!

Algorithm 3 (Rainbow Band Separation). Reconciliation may be extended for a Rainbow scheme where the final stage has o oil and $v=n-o$ vinegar variables (which has the smaller indices):

1. Perform basis change $w_{i}:=w_{i}^{\prime}-\lambda_{i} w_{n}^{\prime}$ for $i=1 \cdots v, w_{i}=w_{i}^{\prime}$ for $i=$ $v+1 \cdots n$. Evaluate $\mathbf{z}$ in $\mathbf{w}^{\prime}$.

2. Find $m$ equations by setting all coefficients of $\left(w_{n}^{\prime}\right)^{2}$ to be zero; there are $v$ variables in the $\lambda_{i}$ 's.

3. Set all cross-terms involving $w_{n}^{\prime}$ in $\mathbf{z}_{1}-\sigma_{1}^{(1)} \mathbf{z}_{v+1}-\sigma_{2}^{(1)} \mathbf{z}_{v+2}-\cdots-\sigma_{o}^{(1)} \mathbf{z}_{m}$ to be zero and find $n-1$ more equations. Note that $\left(w_{n}^{\prime}\right)^{2}$ terms are assumed gone already, so we can no longer get a useful equation.

4. Solve $m+n-1$ quadratic equations in $o+v=n$ unknowns. We may use any method (e.g., $\mathbf{F}_{\mathbf{4}}$ or $\left.X L\right)$.

5. Repeat the process to find $P_{n-1}$. Now set $w_{i}^{\prime}:=w_{i}^{\prime \prime}-\lambda_{i} w_{n-1}^{\prime \prime}$ for $i=1 \cdots v$, and set every $\left(w_{n-1}^{\prime \prime}\right)^{2}$ and $w_{n}^{\prime \prime} w_{n-1}^{\prime \prime}$ term to zero after making the substitution. Also set $\mathbf{z}_{2}-\sigma_{1}^{(2)} \mathbf{z}_{v+1}-\sigma_{2}^{(2)} \mathbf{z}_{v+2}-\cdots-\sigma_{o}^{(2)} \mathbf{z}_{m}$ to have a zero secondto-last column. This time there are $2 m+n-2$ equations in $n$ unknowns.

6. Continue in the same vein to find $P_{n-2}, \ldots, P_{v+1}$.

The idea was mentioned by Mr. Yu-Hua Hu to one of the authors in a conversation, for which we are indebted. And this attack explains why the current parameter set suggested looks like that in Sec. 5 .

Example 8. We run the attack on an instance of enTTS $(16,22)$ [25] which has the shape $(6,7,1,1,7)$. The algebraic portion of the attack results in a system with 22 variables and 37 equations. This with XL at degree $D_{X L}=6$ can be solved using 400MB (actually 415,919,856 bytes) of memory and 123,257 seconds on a 16-core, $2.2 \mathrm{GHz}$ Opteron machine with a total of 1,877,572 seconds of K8-CPU time. The number of multiplications is about $2^{47}$, or $\sim 16$ cycles a multiplication.

On a single core, a K8 machine running XL-Wiedemann can average one multiplication in $\mathrm{GF}\left(2^{8}\right)$ in about 9 cycles. The slowdown comes from the communications requirement between cores.

Example 9. The attack on an instance of enTTS(20,28) 25] should result in a system with 22 variables and 37 equations. This with XL at degree $D_{X L}=7$ should be solvable in $15 \mathrm{~GB}$ of main memory and about $2^{56}$ multiplications. This is under the design complexity of $2^{72}$.

We are also testing the prowess of other system-solving methods like Magma's $\mathbf{F}_{\mathbf{4}}$.

\subsection{Interlinked/Accumulating Kernels and MinRank}

As noted in 25] and recapped in Sec. 3] if $\mu$ combinations of central equations stays at the minrank, a Rank attack often speed up $\mu$-fold, and which is termed interlinking or accumulation of kernels. 
Recently Billet and Gilbert [4] cryptanalyzed the Rainbow instance of [7] in $2^{64} 3 \mathrm{DES}$ unit times (they stated $2^{71}$, but GF(256)-multiplications is a very small unit; NESSIE for example counted 3DES units) using the same principle. While we exhibit a faster attack on that rainbow instance above, the same extended accumulating-kernel minrank attack is more widely applicable:

Proposition 3 (Billet-Gilbert). Kernels of the initial block of equations in a rainbow-like multivariate always accumulate such that any vector in $\mathbf{x}$-space with the initial vinegar components all vanishing has at least a $1 / q$ probability of being found by the MinRank attack.

Example 10. We can cryptanalyze enTTS $(20,28)\left[25\right.$ in $2^{64}$ via the accumulating kernels attack.

In fact, this pitfall is sometimes easy to overlook:

Proposition 4. We can cryptanalyze TRMS from [20] in $\sim 2^{62}$ via the accumulating kernels attack.

Proof. The central map has this piece with $*_{3}$ meaning multiplication in $\operatorname{GF}\left(2^{24}\right)$ :

$$
\left(\begin{array}{l}
y_{17} \\
y_{18} \\
y_{19}
\end{array}\right)=\left(\begin{array}{l}
x_{17} \\
x_{18} \\
x_{19}
\end{array}\right) *_{3}\left(\begin{array}{c}
x_{8} \\
x_{9}+x_{11}+x_{12} \\
x_{13}+x_{15}+x_{16}
\end{array}\right)+\left(\begin{array}{c}
c_{29} x_{4} x_{16} \\
c_{30} x_{5} x_{10} \\
c_{31} x_{15} x_{16}
\end{array}\right)+\left(\begin{array}{c}
c_{32} x_{9} \\
c_{33} x_{10} \\
c_{34} x_{11}
\end{array}\right) .
$$

Each of these equations are only of rank 8 (the minrank) in GF(256), and the $y_{17}$ and $y_{19}$ form a pair of equations that has $q=256$ interlinked kernels. Evaluating as in Sec. 3 gives $\approx 2^{62}$.

In our schemes, the attack has complexity roughly $q$ to the number of equations in the first block times change, which comes out to about $2^{85}, 2^{100}, 2^{118}$.

\subsection{The Challenge}

From all the above, we can see that we need to be very careful in our design of the parameter for Rainbow like schemes.

Proposition 5. To build a scheme with design security $C$ over the base field $\operatorname{GF}(q)$, we let $\ell$ be the smallest integer such that $q^{\ell+1} \gtrsim C$, then:

- The initial segment must contain $\ell-1$ or more vinegar variables. The final segment must contain $\ell-1$ or more equations and exactly as many as there are total vinegar variables.

- There should be enough equations to avoid direct solution via a LazardFaugère solver.

Current estimate 24] is that 20 underdetermined equations in $\mathrm{GF}\left(2^{8}\right)$ achieves $2^{72}$; 24 equations achieves $2^{82}$; each extra equation roughly gives a factor $\gtrsim 2^{2.5}$ to the complexity [24]. 
We conclude that all three Rainbow like schemes we propose below have security levels above $2^{80}$ elementary operations. The best attack is with Algorithm 3 and the expected complexity in $\operatorname{GF}\left(2^{8}\right)$ multiplications is $2^{84}, 2^{87}, 2^{80}$ respectively.

1. Rainbow $(20,10,4,10)$, in the extended form $((15,10),(4,4),(1,10))$

2. Rainbow $(18,12,12)$, in the extended form $((17,12),(1,12))$

3. Rainbow $(20,14,14)$, in the extended form $((19,14),(1,14))$.

Of course, without using the extended form, the security level would not be any lower, the extended form merely guarantees the existence of a signature always.

We hasten to add that the form given above is not much slower in signing than the previous TTS. In preliminary runs, a single signature for $(20,10,4,10)$ version averages to about $157 \mu \mathrm{s}$, still way faster than any competitor.

\section{Conclusion}

In this paper, we present a new differential attack and a new Rainbow constructions. We design new schemes for practical applications.

With these constructions, we note that the design security of the system would still go up exponentially as the length of the hash in both generic (rainbow) and sparse (TTS) variants. Perhaps, we might even say that the kinks of this approach is being ironed out, and multivariate cryptographers are finally beginning to understand Rainbow-like Multivariate Signatures.

Another development that affects Rainbow-like schemes is the fact that SHA-1 is being phased out in the wake of recent results [21. This means that hashes and hence signatures might become longer in a hurry. ECC is affected in much the same way, because 163- or 191-bit ECC may be obsoleted when everyone switches to SHA-2 (no one really wants to use a truncated hash if it can be helped). Even such state-of-the-art work as 3 would force the slightly uncomfortable SHA224. With multivariate signature schemes, an additional problem is the large (and sometimes redundant, cf. [23]) keys. One might look toward other base fields such as GF(16) to help with the key size problem, but this would also pose new challenges in optimization. Another way is to look for a safe TTS (built on the similar layer structures as specified above), now that hash sizes has gotten longer. Though the new attacks are found on Rainbow schemes, these attacks can be easily prevented by adjusting the parameter. All in all, we think that multivariates including Rainbow-like schemes still deserve a good look as the age of quantum computers approaches.

\section{Acknowledgements}

JD and BY are grateful to the Humboldt and Taft Foundations, and the Taiwan Information Security Center [National Science Council Project NSC 96-2219-E011-008 / NSC 96-2219-E-001-001] without whose valuable support much of this work would not have been possible. BY would also like to thank NSC for partial sponsorship on Project NSC 96-2623-7-001-005-D. 
Comments and correspondence: Please address to BY at by@moscito.org.

\section{References}

1. Bardet, M., Faugère, J.-C., Salvy, B.: On the complexity of Gröbner basis computation of semi-regular overdetermined algebraic equations. In: Proceedings of the International Conference on Polynomial System Solving, pp. 71-74 (2004); Previously INRIA report RR-5049

2. Bardet, M., Faugère, J.-C., Salvy, B., Yang, B.-Y.: Asymptotic expansion of the degree of regularity for semi-regular systems of equations. In: Gianni, P. (ed.) MEGA 2005, Sardinia (Italy) (2005)

3. Bernstein, D.J.: Curve25519: New diffie-hellman speed records. In: Yung, M., Dodis, Y., Kiayias, A., Malkin, T.G. (eds.) PKC 2006. LNCS, vol. 3958, pp. 207228. Springer, Heidelberg (2006)

4. Billet, O., Gilbert, H.: Cryptanalysis of rainbow. In: De Prisco, R., Yung, M. (eds.) SCN 2006. LNCS, vol. 4116, pp. 336-347. Springer, Heidelberg (2006)

5. Coppersmith, D., Stern, J., Vaudenay, S.: The security of the birational permutation signature schemes. Journal of Cryptology 10, 207-221 (1997)

6. Courtois, N.T., Klimov, A., Patarin, J., Shamir, A.: Efficient algorithms for solving overdefined systems of multivariate polynomial equations. In: Preneel, B. (ed.) EUROCRYPT 2000. LNCS, vol. 1807, pp. 392-407. Springer, Heidelberg (2000); Extended Version: http://www.minrank.org/xlfull.pdf

7. Ding, J., Schmidt, D.: Rainbow, a new multivariable polynomial signature scheme. In: Ioannidis, J., Keromytis, A.D., Yung, M. (eds.) ACNS 2005. LNCS, vol. 3531, pp. 164-175. Springer, Heidelberg (2005)

8. Dubois, V., Fouque, P.-A., Shamir, A., Stern, J.: Practical cryptanalysis of sflash. In: Menezes, A. (ed.) CRYPTO 2007. LNCS, vol. 4622, pp. 1-12. Springer, Heidelberg (2007)

9. Faugère, J.-C.: A new efficient algorithm for computing Gröbner bases $\left(F_{4}\right)$. Journal of Pure and Applied Algebra 139, 61-88 (1999)

10. Faugère, J.-C.: A new efficient algorithm for computing Gröbner bases without reduction to zero $\left(F_{5}\right)$. In: International Symposium on Symbolic and Algebraic Computation — ISSAC 2002, pp. 75-83. ACM Press, New York (2002)

11. Faugère, J.-C., Perret, L.: Polynomial equivalence problems: Algorithmic and theoretical aspects. In: Vaudenay, S. (ed.) EUROCRYPT 2006. LNCS, vol. 4004, pp. 30-47. Springer, Heidelberg (2006)

12. Garey, M.R., Johnson, D.S.: Computers and Intractability - A Guide to the Theory of NP-Completeness. W.H. Freeman and Company (1979) ISBN 0-7167-1044-7 or $0-7167-1045-5$

13. Geiselmann, W., Steinwandt, R., Beth, T.: Attacking the affine parts of SFlash. In: Honary, B. (ed.) Cryptography and Coding 2001. LNCS, vol. 2260, pp. 355359. Springer, Heidelberg (2001); Extended version http://eprint.iacr.org/ 2003/220/

14. Goubin, L., Courtois, N.T.: Cryptanalysis of the TTM cryptosystem. In: Okamoto, T. (ed.) ASIACRYPT 2000. LNCS, vol. 1976, pp. 44-57. Springer, Heidelberg (2000)

15. Joux, A., Kunz-Jacques, S., Muller, F., Ricordel, P.-M.: Cryptanalysis of the tractable rational map cryptosystem. In: PKC [19], pp. 258-274. 
16. Kipnis, A., Patarin, J., Goubin, L.: Unbalanced Oil and Vinegar signature schemes. In: Stern, J. (ed.) EUROCRYPT 1999. LNCS, vol. 1592, pp. 206-222. Springer, Heidelberg (1999)

17. Kipnis, A., Shamir, A.: Cryptanalysis of the oil and vinegar signature scheme. In: Krawczyk, H. (ed.) CRYPTO 1998. LNCS, vol. 1462, pp. 257-266. Springer, Heidelberg (1998)

18. Patarin, J., Goubin, L.: Trapdoor one-way permutations and multivariate polynomials. In: Han, Y., Quing, S. (eds.) ICICS 1997. LNCS, vol. 1334, pp. 356-368. Springer, Heidelberg (1997); Extended Version http://citeseer.nj.nec.com/ patarin97trapdoor.html

19. Vaudenay, S. (ed.): PKC 2005. LNCS, vol. 3386. Springer, Heidelberg (2005)

20. L.-C. Wang, Y.-H. Hu, F. Lai, C.y. Chou, and B.-Y. Yang. Tractable rational map signature. In PKC [19], pp. 244-257. ISBN 3-540-24454-9

21. Wang, X., Yin, Y.L., Yu, H.: Finding collisions in the full sha-1. In: Shoup, V. (ed.) CRYPTO 2005. LNCS, vol. 3621, pp. 17-36. Springer, Heidelberg (2005)

22. Wolf, C., Braeken, A., Preneel, B.: Efficient cryptanalysis of RSE(2)PKC and RSSE(2)PKC. In: Blundo, C., Cimato, S. (eds.) SCN 2004. LNCS, vol. 3352, pp. 294-309. Springer, Heidelberg (2005); Extended version http://eprint.iacr .org/ 2004/237

23. Wolf, C., Preneel, B.: Superfluous keys in $\mathcal{M}$ ultivariate $\mathcal{Q}$ uadratic asymmetric systems. In: PKC [19], pp. 275-287; Extended version http://eprint.iacr.org/ 2004/361/

24. Yang, B.-Y., Chen, J.-M.: All in the XL family: Theory and practice. In: Park, C.s., Chee, S. (eds.) ICISC 2004. LNCS, vol. 3506, pp. 67-86. Springer, Heidelberg (2005)

25. Yang, B.-Y., Chen, J.-M.: Building secure tame-like multivariate public-key cryptosystems: The new TTS. In: Boyd, C., González Nieto, J.M. (eds.) ACISP 2005. LNCS, vol. 3574, pp. 518-531. Springer, Heidelberg (2005)

26. Yang, B.-Y., Chen, O.C.-H., Bernstein, D.J., Chen, J.-M.: Analysis of QUAD. In: Biryukov, A. (ed.) FSE 2007. LNCS, vol. 4593, pp. 290-307. Springer, Heidelberg (2007) 